\title{
Assessing and Improving Robustness of Psychological Research Findings in Four Steps
}

Dr. Michèle B. Nuijten

\section{Acknowledgements}

The preparation of this chapter was supported by a Veni grant (no. 11507) from the Dutch Research Council.

\section{Abstract}

Increasing evidence indicates that many published findings in psychology may be overestimated or even false. An often-heard response to this "replication crisis" is to replicate more: replication studies should weed out false positives over time and increase robustness of psychological science. However, replications take time and money - resources that are often scarce. In this chapter, I propose an efficient alternative strategy: a four-step robustness check that first focuses on verifying reported numbers through reanalysis before replicating studies in a new sample. 
Nuijten, M. B. (accepted chapter). Assessing and improving robustness of psychological research findings in four steps. In W. O'Donohue, A. Masuda, and S. Lilienfeld (Eds.), Clinical psychology and questionable research practices. Springer.

\section{Introduction}

\section{The Replication Crisis}

Around 2012, scientists started speaking of a "replication crisis" in psychology (Pashler \& Harris, 2012; Pashler \& Wagenmakers, 2012). A growing number of published psychological findings did not seem to hold up when the study was done again in a new sample (e.g., Chabris et al., 2012; Doyen et al., 2012; LeBel \& Campbell, 2013; Matthews, 2012; Pashler et al., 2013). Already since the 1950s, research consistently shows that over $90 \%$ of published psychology papers find support for their main hypothesis (Fanelli, 2010; Sterling, 1959; Sterling et al., 1995), whereas this is virtually impossible given the generally low statistical power in the field (Bakker et al., 2012; Francis et al., 2014). In other words, it seems that many published findings in psychology are too good to be true.

A possible explanation for the excess of positive results in psychology is a combination of publication bias, publication pressure, and questionable research practices (QRPs). Publication bias occurs when articles with statistically significant results have a higher probability of being published than articles with non-significant results (Greenwald, 1975). When researchers experience the pressure to publish (and they often do; Dalen \& Henkens, 2012; Tijdink et al., 2014), publication bias provides a direct incentive to only report positive, significant results. This may cause researchers to (consciously or unconsciously) exploit the inherent flexibility in data collection, processing, and analysis until they find the desired result. When only the "successful" strategy is then reported, one could speak of QRPs (Gelman \& Loken, 2013; Kerr, 1998; Simmons et al., 2011). Evidence from surveys and from comparisons of research plans with the resulting publications seemed to indicate a high prevalence of such QRPs in the psychological literature (Franco et al., 2016; Agnoli et al., 2017; John et al., 2012; but see Fiedler \& Schwarz, 2016). 
Nuijten, M. B. (accepted chapter). Assessing and improving robustness of psychological research findings in four steps. In W. O'Donohue, A. Masuda, and S. Lilienfeld (Eds.), Clinical psychology and questionable research practices. Springer.

\section{Suggested Solution: More Replications}

One reaction to the replication crisis is the call to perform more replication studies. See for example the suggestion to require researchers to perform replication studies in their research area in proportion to the number of original studies they conduct (LeBel, 2015) or to require undergraduate, graduate, or PhD students to perform replication studies (Frank \& Saxe, 2012; Kochari \& Ostarek, 2018). Journals have also taken up this sentiment by actively encouraging authors to publish replication studies (Registered Replication Reports; Association for Psychological Science, n.d.; Jonas et al., 2017; Nosek \& Lakens, 2014), and even funders have set funds aside specifically for replication research (The Dutch Research Council, n.d.). We have also seen an increase in the number of large-scale, multi-lab replication attempts, in which sometimes dozens of labs across the world set out to replicate the same study (e.g., Alogna et al., 2014; R. A. Klein et al., 2014; ManyBabies Consortium, 2020; Moshontz et al., 2018) in order to give a (somewhat) definitive answer to a certain research question.

One of the most well-known examples of a multi-lab replication project is the Reproducibility Project: Psychology, a project with 270 contributing authors led by Prof. Brian Nosek (Open Science Collaboration, 2012, 2015). The goal of the project was to estimate the replicability of psychology by systematically replicating a selection of 100 psychology studies published in prominent journals. They found that the mean effect size in the replication studies was much lower than the mean effect size of the original studies (replication: $r=0.197, \mathrm{SD}=0.257$; original: $r=0.403$, $S D=0.188)$. Furthermore, where the original studies found statistically significant results in $97 \%$ of the cases, only $36 \%$ of the replications did. There is some debate about how exactly these results should be interpreted (C. J. Anderson et al., 2016; see, e.g., Bavel et al., 2016; Etz \& Vandekerckhove, 2016; Gilbert et al., 2016), but the results are generally taken as a sign that the replicability rate in psychology might be low. 
Nuijten, M. B. (accepted chapter). Assessing and improving robustness of psychological research findings in four steps. In W. O'Donohue, A. Masuda, and S. Lilienfeld (Eds.), Clinical psychology and questionable research practices. Springer.

The call to replicate more is perfectly sensible and in line with the notion that replication is a cornerstone of science (Lakatos \& Musgrave, 1970; Meehl, 1990). Already in introductory research methods classes, it is generally taught that a single study cannot provide definitive answers to a research question (Morling, 2020, p. 14). Instead, multiple studies need to convincingly show an effect before we continue to build on it any further. Unfortunately, the psychological literature does not seem to reflect this notion. Even though the literature contains many conceptual replications that investigate the boundaries and generalizability of a theory (Neuliep \& Crandall, 1993), direct replication studies that are mainly aimed at checking whether an effect is robust in the first place seem to be rare (Makel et al., 2012). Without direct replications, researchers would have to rely on individual studies to build their work upon. Unfortunately, the replication crisis has shown us that the findings in many of these individual studies may be false positives. This means that many researchers may be trying to build research lines based on dead ends. By encouraging more replication studies, we could ideally weed out many of the false positives and the foundation to build upon would be stronger.

Even though encouraging replications could in theory help in assessing and improving robustness of published findings, there is a significant disadvantage: performing a replication study takes considerable time and money; resources that are usually scarce. In this chapter, I would like to suggest a more efficient way to assess whether a published result is robust through a four-step robustness check that first focuses on verification of reported numbers through reanalysis before replicating a study in a new sample.

\section{Statistical Reproducibility is a Prerequisite for Replication}

When replicating a study, researchers are often interested in comparing the results of their replication with those of the original study. There is no consensus on the best way to decide whether or not the original finding has been replicated (Open Science Collaboration, 2015; Zwaan et al., 2017), but in general the main statistical results from the original study are compared to the main 
Nuijten, M. B. (accepted chapter). Assessing and improving robustness of psychological research findings in four steps. In W. O'Donohue, A. Masuda, and S. Lilienfeld (Eds.), Clinical psychology and questionable research practices. Springer.

statistical results from the replication. If the two sets of results are similar enough, one could conclude that the original study has successfully been replicated, and if the two sets of results lie too far apart, one could conclude that the original study was not successfully replicated. One key point to keep in mind here is that, for this comparison to be meaningful, the reported numbers have to be correct: the reported results should not contain typos, calculation errors, or other mistakes. In other words, the reported results should be statistically reproducible: reanalysis of the original data following the reported procedures should result in the same numbers as those reported in the paper (Nuijten et al., 2018).

Roughly, there can be two reasons why a result is not statistically reproducible (Nosek et al., 2021). First, a process reproducibility failure occurs when it is not possible to repeat the steps of the original analysis, for example because of unavailable data or code, unclear description of the analysis steps, or unavailable software or tools. In psychology, raw data have been notoriously unavailable (Wicherts et al., 2006), although there has been some improvement in recent years (Hardwicke et al., 2018; Kidwell et al., 2016; Nuijten, Borghuis, et al., 2017). Furthermore, even when raw data are available, there are often insufficient details reported to redo the original analysis (e.g., Kidwell et al., 2016). For example, the data are insufficiently documented (e.g., instead of informative variable names, variables still have SPSS' default labels VAR0001, VAR0002 etc.), or the paper only states that "an ANOVA" has been done, without elaborating on any data preprocessing steps (e.g., the removal of outliers) or specific details about the analysis.

Second, an outcome reproducibility failure occurs when the original analysis can be repeated but leads to a different result than the one that is reported (Nosek et al., 2021). In general, it is plausible to assume that reanalyzing the same data according to the same methods leads to the same results. Unfortunately, this is not always the case. For example, two recent studies reran the original analyses on the original data of a set of psychology studies and compared the outcomes with the reported results. Both studies found numerical discrepancies in over $60 \%$ of the reanalyzed 
Nuijten, M. B. (accepted chapter). Assessing and improving robustness of psychological research findings in four steps. In W. O'Donohue, A. Masuda, and S. Lilienfeld (Eds.), Clinical psychology and questionable research practices. Springer.

studies (Hardwicke et al., 2018, 2020). Furthermore, evidence from over 16,000 psychology papers showed that roughly half of the papers contained at least one $p$-value that was not consistent with the reported test statistic and degrees of freedom. In roughly one in eight articles, the recomputed $p$-value was not significant whereas the reported $p$-value was, or vice versa (Nuijten et al., 2016).

Statistical reproducibility of results is a basic, necessary requirement for scientific quality (Chambers, 2020; Peng, 2011). If a reported result cannot be linked back to the underlying data, it is extremely difficult (if not impossible) to meaningfully interpret that result. As such, if an investigator wants to know whether a certain result is robust or not, that investigator may not need to perform a full replication study: if the result is not statistically reproducible, it is not robust. I would therefore like to argue that verifying reported results should be the first step in assessing the robustness of a result (see also LeBel et al., 2018; Nuijten et al., 2018; Stark, 2018). To facilitate such statistical reproducibility checks, I suggest a practical four-step approach.

\section{Checking Robustness of a Finding in Four Steps}

\section{Step 1: Check for internal inconsistencies in reported statistics}

A first step when checking if a result is robust is to check if the reported statistics are internally consistent. To complete this step, you do not need access to the raw data, you only need the reported statistical results. A statistical reporting inconsistency occurs when a set of numbers that belongs together does not match. For example, consider the following sentence: " $70 \%$ of patients recovered within three months after the first diagnosis $(65 / 100)^{\prime \prime}$. Only by looking at the reported numbers in this sentence, we can already see that something is wrong: $65 / 100$ is $65 \%$, not 70\%. At this point, it is unclear which of the reported numbers is incorrect, but what is clear, is that this set of numbers presents an impossible combination and can therefore not have come from the underlying raw data. In other words: this result is not statistically reproducible. 
Nuijten, M. B. (accepted chapter). Assessing and improving robustness of psychological research findings in four steps. In W. O'Donohue, A. Masuda, and S. Lilienfeld (Eds.), Clinical psychology and questionable research practices. Springer.

Internal inconsistencies could be detected in a wide range of statistics. Other than percentages that have to match the accompanying fractions, examples are:

- Reported total sample sizes should match the subgroup sizes

- Reported effect estimates should fall within the bounds of the accompanying confidence interval

- Reported odds ratios should match the accompanying frequency table

- Reported sensitivity of a diagnostic test should match the true/false positive and true/false negative rates

- Reported t- and F-values should match the reported means and standard deviations

- Reported $p$-values should match the test statistic and degrees of freedom

Currently, several tools and algorithms are being developed to automatically (or semiautomatically) detect statistical reporting inconsistencies. One of these tools is statcheck: a free $\mathrm{R}$ package (Epskamp \& Nuijten, 2014) and accompanying web app (http://statcheck.io; Rife et al., 2016) that automatically extracts Null Hypothesis Significant Tests (NHST) results from articles and recomputes $p$-values based on the reported test statistic and degrees of freedom. For example, say that an article reports the following sentence: "We found that the treatment group scored significantly higher on well-being than the control group, $t(28)=1.46, p<.05$." If you would scan this article with statcheck, it would recognize the reported $t$-test and use the reported test statistic (1.46) and degrees of freedom (28) to recompute the $p$-value. In this case, the recomputed $p$-value would be 0.155 . This is not consistent with the reported $p$-value of $<.05$. What is more in this case is that the reported result would be flagged as a decision inconsistency (also sometimes called a gross inconsistency or gross error): based on the recomputed $p$-value one would draw a different conclusion (i.e., the difference between groups is not significant). Statcheck currently only recognizes statistics reported in APA style (American Psychological Association, 2019), which makes 
Nuijten, M. B. (accepted chapter). Assessing and improving robustness of psychological research findings in four steps. In W. O'Donohue, A. Masuda, and S. Lilienfeld (Eds.), Clinical psychology and questionable research practices. Springer.

the tool primarily useful for psychology papers. Also please see Nuijten et al. (2017) for a full analysis of statcheck's accuracy in spotting (decision) inconsistencies.

Another example of a tool to spot statistical reporting inconsistencies is the tool GRIM (granularity-related inconsistency of means; Brown \& Heathers, 2017). GRIM can spot whether reported means that are based on integer data (e.g., from Likert-type scales) are possible in combination with a certain sample size and total number of items. Other examples include an algorithm to check whether reported effect sizes match their confidence intervals and $p$-values (Georgescu \& Wren, 2018) and a semi-automated protocol to assess inconsistencies in a wide range of statistics (van Aert et al., 2021).

In sum, regardless of whether one can automate the process of looking for inconsistencies in statistical reporting or not, these above-mentioned tools offer relatively quick procedures that do not require access to anything else but the paper itself. This makes it an efficient first "sanity check" in assessing whether or not a reported result is robust.

\section{Step 2: Reanalysis of original data}

A second step when checking if a result is robust is to reanalyze the original data according to the reported procedure to see if one can find the same results as reported. As opposed to step 1, one now does need access to the original data and information about the original analysis.

There is not one way to approach a reanalysis, but there are some general steps one could follow. First, determine whether the raw data underlying the finding of interest are available. A quick way to do so is to search for a Data Availability Statement (a standardized short statement about whether data are available, requested by an increasing number of journals, incl. e.g., PLOS journals) or an Open Data Badge (a badge printed at the top of the paper that signals that data are available, used by an increasing number of psychology journals, incl. APS and APA journals; Center for Open Science, n.d.-a). If data are stated to be available, it is unfortunately still not a guarantee that they 
Nuijten, M. B. (accepted chapter). Assessing and improving robustness of psychological research findings in four steps. In W. O'Donohue, A. Masuda, and S. Lilienfeld (Eds.), Clinical psychology and questionable research practices. Springer.

actually are. For example, 25-30\% of articles published in Frontiers in Psychology and in several PLOS journals that stated that data were available, did not contain or link to the raw data (Chambers, 2017, p. 86; Nuijten, Borghuis, et al., 2017). Of course, it is also possible to contact the original authors to ask for the data and other relevant materials, although historically, this is often not very successful (Wicherts et al., 2006).

Next, download the data and try to open the file. Ideally, raw data files are in a (relatively) standard format, such as .csv, .txt, .xlsx, or .sav, and one can open the data without needing expensive software. Once one can open the data, it is important to skim through the file to see whether the data seem to be understandable and complete (i.e., are all variables mentioned in the paper also mentioned in the data? Does the number of rows correspond to the reported number of participants?). This step includes determining whether the authors also shared a codebook that (among other things) explains all variables in the data and their values, how missing data are coded, and whether any data preprocessing steps have already taken place (e.g., reverse coding of contraindicative items).

Beside the data, one also needs as much information as possible about the original data analysis. Therefore, a good next step, once one has access to the data and understand the file, is to determine whether the authors also shared their analysis script. An analysis script is preferable over the analysis description in the methods or results section of the paper, because a script usually contains more explicit and detailed information about the subsequent steps in the analysis. If an analysis script is not available, extract as much detail about the data preprocessing and analysis from the paper (and supplementary files) as possible.

Once an investigator has all available data and information about the analysis, the reanalysis phase itself can begin. Here, the investigator has to decide which reported values you want to try and reproduce: this could be all the reported numbers in the paper or only some key values related to the main conclusion, or something in between. In this reanalysis phase, follow the original 
Nuijten, M. B. (accepted chapter). Assessing and improving robustness of psychological research findings in four steps. In W. O'Donohue, A. Masuda, and S. Lilienfeld (Eds.), Clinical psychology and questionable research practices. Springer.

analysis steps themselves as closely as possible, where possible using the same software, version, and operating system.

How much time it takes to reanalyze original data according to the original procedure depends for a large part on the complexity of the data and the analysis, and on the clarity with which the original procedure was reported. For example, in one reanalysis project, the researchers spent between 1 and 30 hours (median $=7$ ) on each reproducibility check (Hardwicke et al., 2020). Roughly speaking, a reanalysis will likely take more time than a check for statistical reporting inconsistencies in the paper, but less time than a full replication study.

\section{Step 3: Sensitivity checks}

Even if one were able to reproduce the same results through reanalysis of the original data, there is no guarantee that the result is robust. Therefore, a third step when checking if a result is robust is to reanalyze the original data using slightly different but still justifiable preprocessing and analysis steps than the ones reported. This can shed light on whether the result is robust to alternative choices.

Say that an investigator followed the reported analytical procedures and that she was able to reproduce the main result from the original data. However, it could be the case that the original authors removed an outlier in their analysis. It may also be the case that if she does not remove this outlier, the result changes substantively. Similarly, it may happen that the removal of a seemingly arbitrary covariate can make the result disappear. And what if other ways to construct the final variables of interest lead to different results? There are many choices involved in data preprocessing and statistical analysis, and if only a very specific combination of analytical steps leads to a significant result, one may question its robustness (Gelman \& Loken, 2013).

It is difficult to provide general instructions for how to do such sensitivity checks, because the set of justifiable analytical choices is highly dependent on the specific research question, type of 
Nuijten, M. B. (accepted chapter). Assessing and improving robustness of psychological research findings in four steps. In W. O'Donohue, A. Masuda, and S. Lilienfeld (Eds.), Clinical psychology and questionable research practices. Springer.

study, available variables, and other contextual factors. That said, there are several general questions one could ask that could guide the sensitivity checks (see also Patel et al., 2015; Steegen et al., 2016).

First, one could consider the data preprocessing steps that led to the final data on which the analyses were performed. For example, if the original authors used questionnaire data, how did they summarize the scores on individual items to a score on the construct of interest? In case not all items were included, what happens if one does include them all? What if instead of calculating a sum score over the items, one calculates a factor score? If the authors turned a continuous variable into a categorical one (e.g., classifying BMI into underweight, normal weight, overweight, or obese), would other cut-offs to determine the categories also be justifiable? Yet other questions related to data preprocessing could concern inclusion/exclusion criteria; were participants excluded from the final analysis? What happens if one does include them or use slightly different exclusion criteria?

A second type of question one could ask is to what extent a finding is sensitive to different choices in the analysis itself. First, check if the authors did a correct analysis in the first place (e.g., including an interaction effect in the analysis, instead of erroneously comparing the p-values of two different effects; Nieuwenhuis et al., 2011). But also within a correct analysis, different choices can be made. This could include questions, such as: how did the authors deal with missing values and would another strategy be justifiable as well? Which (if any) covariates were included in the analysis, and would another selection be equally justifiable? In the case of frequentist hypothesis testing, one could consider adding/removing corrections for multiple testing, trying other cut-offs (such as the often arbitrary a significance level of $p<.05)$, choices for one-tailed testing, or comparisons with the outcome of Bayesian hypothesis testing. 
Nuijten, M. B. (accepted chapter). Assessing and improving robustness of psychological research findings in four steps. In W. O'Donohue, A. Masuda, and S. Lilienfeld (Eds.), Clinical psychology and questionable research practices. Springer.

\section{Step 4: Replication in a new sample}

A fourth and final step when checking if a result is robust could be to perform a replication study in a new sample (Nuijten et al., 2018). Replications come in many shapes and sizes, but they are usually classified along a continuum ranging from a direct replication (also known as exact replication or close replication) to a conceptual replication (LeBel et al., 2017). In a direct replication, the methods of the original study are followed as closely as possible. The results of a direct replication (or preferably multiple direct replications) can be used to assess the reliability of a result: will the effect (or lack thereof) show up again if we repeat a study? Conceptual replications on the other hand, aim to test the generalizability of a result by testing the original hypothesis using different methods than the original study (e.g., in a different setting, in a different population, or using different operationalizations). Arguably, it makes most sense to start with direct replications to first rule out (at least to some extent) that an original result is not a false positive or false negative, before setting out to check generalizability of a result that might turn out to be a fluke (Zwaan et al., 2017).

A lot has been written about ways to conduct (or evaluate) a good direct replication study (e.g., Brandt et al., 2014; LeBel et al., 2019), and in this chapter I mainly want to focus on the lessdiscussed statistical reproducibility checks. However, there are some general guidelines when doing a direct replication study that one can take into account. Generally speaking, a direct replication aims to study the same effect as the original study and should follow the original study's procedures as closely as possible, barring some inevitable differences (e.g., the actual participants or the point in time that the studies take place). It is also advised to have high statistical power, which often means significantly increasing the sample size as compared to the original study (S. F. Anderson \& Maxwell, 2017; Open Science Collaboration, 2015). Furthermore, several methods have been proposed to interpret the results of the replication study compared to the original study, including subjective evaluation, comparing $p$-values, effect sizes, confidence intervals, Bayes factors, and more (Open 
Nuijten, M. B. (accepted chapter). Assessing and improving robustness of psychological research findings in four steps. In W. O'Donohue, A. Masuda, and S. Lilienfeld (Eds.), Clinical psychology and questionable research practices. Springer.

Science Collaboration, 2015; Simonsohn, 2015; Verhagen \& Wagenmakers, 2014; Zwaan et al., 2017). Note that several recommendations to improve the quality of replications also hold for original studies, such as transparent reporting, high statistical power, robust statistical methods, and sharing data and materials (Benjamin et al., 2018; Brandt et al., 2014; Lakens et al., 2018; Lakens \& Evers, 2014; LeBel et al., 2019; Munafò et al., 2017; Nosek et al., 2012; Simmons et al., 2011).

\section{Interpreting the outcomes of reproducibility checks}

Strictly speaking, any failure to reproduce a reported result in any of the first three steps of the robustness check would allow one to conclude that a result - as reported - is not robust. However, to what extent this is problematic for an overall conclusion and whether it is still useful to follow subsequent steps of the robustness checks is for a large part context-dependent. For example, it matters (a) if the original authors can help clear up any discrepancies, (b) how big any discrepancies are between the reported and recalculated numbers, (c) how important the result is for the overall conclusion, (d) if a failure to reproduce was due to a process or outcome reproducibility failure, and (e) the overall goal, with which one were doing a robustness check.

\section{Contacting the original authors}

When encountering a reproducibility failure (process or outcome), one can consider contacting the original authors to ask for help and/or clarification. In previous reanalysis studies, original authors were often able to help resolve reproducibility issues (Hardwicke et al., 2018, 2020). Even though it is a positive sign that they were helpful in resolving these specific issues, it is far from ideal if reported results can only be reproduced with the help of the original authors. Especially if one want to assess the robustness and reproducibility of results published several years ago, the authors may not be able to help anymore: they may not have access to the data or scripts themselves anymore, or it may even not be possible to contact them at all. Full statistical 
Nuijten, M. B. (accepted chapter). Assessing and improving robustness of psychological research findings in four steps. In W. O'Donohue, A. Masuda, and S. Lilienfeld (Eds.), Clinical psychology and questionable research practices. Springer.

reproducibility can only be achieved when the published paper and materials contain sufficient information to independently and successfully redo the original analysis.

\section{Size and importance of analytical discrepancies}

The size and context of any outcome reproducibility failure matters. In most cases, a rounding error in the fourth decimal of a $p$-value mentioned in a footnote is probably less consequential than a major discrepancy in the reported and recalculated key outcome of a paper.

There are several ways to judge the size and importance of a reproducibility failure. One option is to look at the difference between the reported and recalculated number. For example, if a reported correlation is .80 , a recalculated correlation of .60 presents a larger discrepancy than a recalculated correlation of .78 (see e.g., Petrocelli et al., 2013). Instead of using absolute differences, you could also look at relative differences, expressed in percentages. If we stick to the same example, the percentage error in the first scenario is equal to $(|.60-.80|) / .80 * 100 \%=25 \%$, and in the second scenario it is equal to $(|.78-.80|) / .80 * 100 \%=2.5 \%$. Earlier research defined a percentage error larger than $10 \%$ as a major numerical error (Hardwicke et al., 2018, 2020).

Another option to classify the size and/or importance of a discrepancy is to look at the statistical decision based on the reported numbers. For example, most of the research in psychology retains a significance level of .05 , meaning that a $p$-value smaller than .05 is considered as statistically significant. Several studies that recalculated $p$-values based on the reported test statistic and degrees of freedom used this cut-off to distinguish between inconsistencies and gross inconsistencies (or errors and decision errors, respectively). If the recalculated $p$-value did not match the reported one, but both were on the same side of the .05 threshold, e.g., reported $p=.03$ vs. recalculated $p=.04$, this was classified as an inconsistency. If a reported $p$-value was statistically significant, but the recalculated $p$-value was not (or vice versa), e.g., reported $p=.03$ vs. recalculated 
Nuijten, M. B. (accepted chapter). Assessing and improving robustness of psychological research findings in four steps. In W. O'Donohue, A. Masuda, and S. Lilienfeld (Eds.), Clinical psychology and questionable research practices. Springer.

$p=.07$, this was classified as a gross inconsistency. (see e.g., Bakker \& Wicherts, 2011; Nuijten et al., 2016).

The location of a statistical result and its importance for the main conclusion can also help in determining the seriousness of a reproducibility failure. With respect to location, results reported in the abstract of a paper can be assumed to be of more importance for a conclusion than results in a footnote or appendix (Georgescu \& Wren, 2018).

Finally, it is also possible to look at the implication of a discrepancy in more depth. In one reanalysis study, the authors concluded that for the studies that contained reproducibility problems (errors in the code and small discrepancies in the number of participants included), the overall conclusions did not change (Naudet et al., 2018).

\section{Process reproducibility failure}

When interpreting a reproducibility failure in one of the first three steps above (checking internal inconsistencies, reanalysis, or sensitivity analyses), it matters whether one encountered a process or outcome reproducibility failure. Remember that a process reproducibility failure occurs when not all steps could be followed to redo the original analysis, whereas an outcome reproducibility failure occurs when the outcome of the reanalysis shows a different result than the one originally reported (Nosek et al., 2021). An outcome reproducibility failure is a more clear-cut outcome than a process reproducibility failure: in the former case one can conclude that a reported result is not robust, whereas in the latter case one cannot assess the robustness of a result.

In case of an outcome reproducibility failure, the reported numbers are not in line with the underlying data and reported analytical method. In such a case, trust in the reported results and possibly also conclusion decreases. However, in case of a process reproducibility failure, it is not possible to verify the reported results. This is problematic, because this means that one just have to "trust" that all reported numbers are correct when interpreting the conclusion, and unfortunately, 
Nuijten, M. B. (accepted chapter). Assessing and improving robustness of psychological research findings in four steps. In W. O'Donohue, A. Masuda, and S. Lilienfeld (Eds.), Clinical psychology and questionable research practices. Springer.

we know from previous research that this may not be the case (Hardwicke et al., 2020, 2020; Nuijten et al., 2016). Furthermore, if the process reproducibility is caused by a lack of access to the raw data (as opposed to unclear analytical steps), it is also not possible to assess to what extent a result is sensitive to alternative analytical choices.

If earlier steps of the four-step robustness check cannot be completed, it may be risky to proceed to step 4 and perform a replication anyway. After all, it is hard to meaningfully compare replication results to the original results, if one does not know if the numbers in the original study are correct in the first place. If and how a process reproducibility failure should affect one's decision whether or not to do a replication study will depend on the reason why one wanted to assess robustness of a finding in the first place.

\section{Reason to assess robustness}

The four-step robustness check could help you decide whether it is worth investing the time and money in performing a replication study. In some cases, one may conclude that if the numbers in the original paper already do not add up, conducting a replication would not be useful. To what extent this holds depends on one's reason to assess the robustness of an original result.

One's goal could be to say something about the robustness of a specific original finding (as opposed to a phenomenon in general). This is often the goal in multi-lab replication projects, such as the Reproducibility Project: Psychology (Open Science Collaboration, 2015). In these cases, the replication studies are often high-powered and pre-registered, arguably enhancing their evidential value compared to the original study they are replicating (Nosek et al., 2021). They therefore attempt to provide a more or less definitive conclusion about the robustness of the original result.

The strict quality controls in these large-scale replication projects are also immediately their disadvantage, however: it is costly to set up such a project, so it is important that the results can be meaningfully compared to the original study. Here, I would argue that following the steps of the 
Nuijten, M. B. (accepted chapter). Assessing and improving robustness of psychological research findings in four steps. In W. O'Donohue, A. Masuda, and S. Lilienfeld (Eds.), Clinical psychology and questionable research practices. Springer.

four-step robustness check can be very valuable to first check statistical reproducibility of the original study and avoid "wasting" resources on a large-scale replication project.

Contrarily, one's goal could also be to get a step closer towards learning the truth about the underlying phenomenon studied in a particular paper. If one then encounters a reproducibility failure in the original study, it does not necessarily have to mean that it is useless to do a replication. It can still be valuable to gather more empirical evidence to answer the original question. However, in such a case, one should be careful when comparing the replication results to the original results. More specifically, one may even want to consider discarding the original study entirely, depending on the severity of the reproducibility failure, and only take the results of the replication study into account to answer the research question.

\section{Successful robustness check}

The sections above are mainly considering scenarios in which the four-step robustness check fails. However, it could of course also be the case that all steps can be completed successfully. First and foremost: this is good news. It means that the reported results are consistently reported, can be traced back to the underlying data, are robust to different analytical choices, and are replicable in new samples. However, passing the four-step robustness is not sufficient to definitively conclude that a result is robust. As with anything in science, it is hard to draw such a black-and-white conclusion at all. Instead, it makes more sense to talk about the degree of robustness or the strength of the evidence that a result is robust.

Some potential problems remain unchecked after following the four-step robustness check. First, the four-step check assumes that the raw data are correct. In other words, the procedure does not take into account errors (or fraud) in data entry. Similarly, these steps do not say anything about the theoretical or methodological quality of a study. For example, if a study uses a biased design and non-validated questionnaires to measure the main constructs, it could still pass all steps in the four- 
Nuijten, M. B. (accepted chapter). Assessing and improving robustness of psychological research findings in four steps. In W. O'Donohue, A. Masuda, and S. Lilienfeld (Eds.), Clinical psychology and questionable research practices. Springer.

step robustness check. Finally, due to sampling error and the probabilistic nature of psychological research, it is possible that two studies find the same results (i.e., the study is successfully replicated), but in both cases the result is a false positive or false negative.

In the end, answering a research question will likely require a long research line comprising of multiple independent studies (incl. direct and conceptual replications) consisting of severe tests (Mayo, 2018), and even then the answer will likely remain tentative (Popper, 1959; O'Donohue, 2021). The decision whether to invest in such a research line as opposed to another, however, can be informed by the success rates of previous robustness checks.

\section{Improving Robustness in Your Own Manuscripts}

The framework of the four-step robustness check is not only useful to assess robustness, but also to improve robustness of a result. Below, I will outline some concrete actions researchers can take that are in line with the logic of the four-step robustness check.

\section{Step 1: Report all relevant statistical information}

It would greatly facilitate robustness checks if relevant statistics were reported in full and with sufficient detail to be able to assess their internal consistency. To illustrate, consider the following conclusion: "All planned contrasts showed support for our hypotheses (all ps $<.05$ )." There is insufficient information here to check whether the reported statistical results are internally consistent. In addition, in this specific case, reporting only $p$-values (and not even the exact $p$-values) also omits important information about effect size and uncertainty in the estimate.

Luckily, many psychology journals require that authors follow the reporting guidelines of the American Psychological Association (APA; American Psychological Association, 2019), which contains specific guidelines on how to report statistical results. For example, they require the following information concerning inferential statistics: "Results of all inferential tests conducted, including exact p-values if null hypothesis statistical testing (NHST) methods were employed, including 
Nuijten, M. B. (accepted chapter). Assessing and improving robustness of psychological research findings in four steps. In W. O'Donohue, A. Masuda, and S. Lilienfeld (Eds.), Clinical psychology and questionable research practices. Springer.

reporting the minimally sufficient set of statistics (e.g., dfs, mean square [MS] effect, MSerror) needed to construct the tests" (Appelbaum et al., 2018; Table 1). In addition, effect size estimates, confidence intervals and other relevant details concerning data preprocessing and analysis should be reported.

\section{Step 2: Provide raw data and analysis scripts}

Sharing data has many benefits (Wicherts, 2013; Wicherts et al., 2012). If raw data are available, others could reanalyze the data to detect potential errors, check robustness of the conclusions to different analytical choices, or even answer entirely new research questions. Ideally, not only the data are shared, but also the original analysis scripts. Methods and results sections in scientific articles often do not contain sufficient detail to rerun an analysis exactly according to the original procedure, whereas analysis code does.

Ideally, data are fully and freely available and well-documented. In other words: the data should be shared according to the FAIR principles (Findable, Accessible, Interoperable, Reusable; Wilkinson et al., 2016). In order to achieve this, authors should not only share the data, but also a detailed codebook that includes information on the variables and other important meta-data, such as where and when the data were collected and under which license the data are shared (for instructions on how to do this, see e.g., Horstmann et al., 2020; O. Klein et al., 2018; Stodden, 2010). Finally, it is important to take privacy legislation into account and protect confidentiality and/or anonymity of the participants.

It is also important to provide an explicit and detailed explanation of the data preprocessing and analysis steps, ideally (again) in the form of an analysis script. Even better would be to share the data and analysis script in a "container" that allows others to rerun the analysis using the same software and operating system as used in the original study (see e.g., O. Klein et al., 2018). 
Nuijten, M. B. (accepted chapter). Assessing and improving robustness of psychological research findings in four steps. In W. O'Donohue, A. Masuda, and S. Lilienfeld (Eds.), Clinical psychology and questionable research practices. Springer.

There can be very good reasons why sharing data is not possible. For example, one may not be allowed to share data because of privacy reasons or because the data are not yours. In such cases, there may be intermediate solutions that are possible. It may for instance be possible to remove identifying data and share only the anonymized part of the data. Or it may be possible to share data with someone else as long as certain agreements not to share the data any further are signed. A more advanced solution includes simulating data that have the same properties as the real data (see also Sweeney, 2002). Running analyses on the simulated data should lead to the same general conclusions as the ones reported (although here a one-on-one comparison of the specific numbers may be difficult). If this is not possible, sharing only part of the data or even only the analysis script without the accompanying data is useful. In sum, when considering the possibilities in sharing data, I encourage authors to focus on what is possible instead of what is not (see also 0. Klein et al., 2018).

\section{Step 3: Perform sensitivity analyses}

Step 3 in assessing robustness entailed running alternative, justifiable analyses to see whether a reported result would still hold up (see above). Such alternative analyses are also known as sensitivity analyses: how sensitive is the result to different analytical approaches? In some scientific fields running sensitivity analyses is a standard part of the research process (e.g., in economics). In psychology, however, this is not yet the case. There are roughly two options to fulfill this step. The first one is to actually perform relevant and justifiable alternative data preprocessing steps and statistical analyses oneself and, importantly, to report all outcomes. Such a multiverse analysis can give insight in how easily one's effect "breaks" under different circumstances, and conversely, how robust it is (Silberzahn et al., 2018; Simonsohn et al., 2019; Steegen et al., 2016). The guidelines in Step 3 of assessing robustness can also be used to list reasonable alternative preprocessing and analytical choices for your own case. 
Nuijten, M. B. (accepted chapter). Assessing and improving robustness of psychological research findings in four steps. In W. O'Donohue, A. Masuda, and S. Lilienfeld (Eds.), Clinical psychology and questionable research practices. Springer.

A second option is to explicitly state that no sensitivity analyses have taken place, for example when an investigator is confident that one's own analysis is the only sensible analysis to perform. It can also help readers evaluate analyses that the investigator conducted and conclusions that the investigator reached if the investigator clearly justify certain analytical choices (e.g., why include a certain covariate but not another?). Such a strategy gains in strength when the analytic plan was preregistered. In a preregistration, the hypotheses, methods, and analysis plans are publicly registered before data collection has taken place. It ensures a clear division between planned, confirmatory analyses and ad-hoc exploratory analyses that may have a higher chance of finding a false positive (Kerr, 1998; Munafò et al., 2017; Simmons et al., 2011; Wagenmakers et al., 2012).

Whether an investigator performs sensitivity analyses or not, being transparent and explicit about analytical choices and the reasoning behind them will help readers to evaluate the results and guide them in any reanalysis they might wish to do.

\section{Step 4: Share study materials}

To facilitate replication of their studies, it is important for researchers to share as many study materials as possible. In theory, a Methods section in a paper should contain sufficient detail to allow for a replication of the study, but in practice this is often not feasible. In order for other researchers to conduct a direct replication, they need to know the exact instruments that were used (e.g., the specific questionnaire), the exact procedure followed (What were the instructions that participants received? In what kind of setting did the study take place?), the exact population that the tested sample was drawn from, etc. This level of detail is usually not accepted in a manuscript (nor beneficial for the readability of a paper), but that does not mean that this information cannot be shared at all. I encourage authors to create extensive supplemental materials for their studies, in which the specific instruments, stimuli, procedural videos, and additional methodological details are shared. 
Nuijten, M. B. (accepted chapter). Assessing and improving robustness of psychological research findings in four steps. In W. O'Donohue, A. Masuda, and S. Lilienfeld (Eds.), Clinical psychology and questionable research practices. Springer.

\section{Concluding Remarks}

In this chapter, I have argued for a four-step robustness check to both assess and improve robustness by first focusing on verifying reported numbers before replicating in a new sample. Statistical reproducibility is a necessary requirement for scientific quality and deserves a place in the spotlight in the current discussions on how to improve psychological science.

Making the four-step robustness check common practice would require both bottom-up and top-down actions. Researchers themselves can use the steps described in this paper to improve robustness of their own work. This is in the best interest of science but can also have direct benefits for the scientists themselves. For example, there is evidence that sharing data is associated with an increased citation rate (Christensen et al., 2019; Piwowar et al., 2007). Researchers can also play a role in studying whether and how the four-step robustness check and other interventions affect robustness of practices and results, ideally by performing randomized controlled trials to study. With respect to top-down actions: several journals already perform checks for internal consistency of submitted manuscripts (e.g., Psychological Science and the Journal of Experimental Social Psychology use the tool statcheck to scan submitted manuscripts for inconsistently reported $p$-values). Furthermore, an increasing number of journals require open data (see e.g., the current signatories to the Transparency and Openness Promotion Guidelines; Center for Open Science, n.d.-b; Chambers, 2018) and accept new article formats focused on rigor and transparency, including Registered Reports (Chambers, 2013), Registered Replication Reports (Association for Psychological Science, n.d.), or Verification Reports (Chambers, 2020).

It is important to keep in mind that while statistical reproducibility is necessary, it is not sufficient for robustness. When determining and/or improving the robustness of a finding, other factors play a role as well. These factors include (but are not limited to) strong theory (Eronen \& Bringmann, 2021), valid measurement (Flake \& Fried, 2020), high statistical power (Button et al., 
Nuijten, M. B. (accepted chapter). Assessing and improving robustness of psychological research findings in four steps. In W. O'Donohue, A. Masuda, and S. Lilienfeld (Eds.), Clinical psychology and questionable research practices. Springer.

2013), robust statistics (Benjamin et al., 2018; Cumming, 2014; Marsman \& Wagenmakers, 2017), severe tests (Mayo, 2018), and transparent reporting (Aczel et al., 2020).

Improving robustness of scientific results is complex and hard work. That said, the scores of initiatives aimed at improving psychological science we have seen in recent years stem hopeful. I would like to close this chapter by encouraging researchers not to get overwhelmed by all these initiatives and practices. If you pick one initiative at a time (e.g., scan a paper with statcheck, share your data, or try out a Bayesian analysis in addition to a traditional frequentist analysis), the robustness of our field will improve. One step at a time. 
Nuijten, M. B. (accepted chapter). Assessing and improving robustness of psychological research findings in four steps. In W. O'Donohue, A. Masuda, and S. Lilienfeld (Eds.), Clinical psychology and questionable research practices. Springer.

\section{References}

Aczel, B., Szaszi, B., Sarafoglou, A., Kekecs, Z., Kucharský, Š., Benjamin, D., Chambers, C. D., Fisher, A., Gelman, A., Gernsbacher, M. A., loannidis, J. P. A., Johnson, E., Jonas, K., Kousta, S., Lilienfeld, S. O., Lindsay, D. S., Morey, C. C., Munafò, M., Newell, B. R., ... Wagenmakers, E.-J. (2020). A consensus-based transparency checklist. Nature Human Behaviour, 4(1), 4-6. https://doi.org/10.1038/s41562-019-0772-6

Agnoli, F., Wicherts, J. M., Veldkamp, C. L. S., Albiero, P., \& Cubelli, R. (2017). Questionable research practices among Italian research psychologists. PLOS ONE, 12(3), e0172792. https://doi.org/10.1371/journal.pone.0172792

Alogna, V. K., Attaya, M. K., Aucoin, P., Bahník, š., Birch, S., Birt, A. R., Bornstein, B. H., Bouwmeester, S., Brandimonte, M. A., Brown, C., Buswell, K., Carlson, C., Carlson, M., Chu, S., Cislak, A., Colarusso, M., Colloff, M. F., Dellapaolera, K. S., Delvenne, J.-F., ... Zwaan, R. A. (2014). Registered Replication Report: Schooler and Engstler-Schooler (1990). Perspectives on Psychological Science, 9(5), 556-578. https://doi.org/10/f6j4g2

American Psychological Association. (2019). Publication manual of the American Psychological Association (7th ed.).

Anderson, C. J., Bahník, Š., Barnett-Cowan, M., Bosco, F. A., Chandler, J., Chartier, C. R., Cheung, F., Christopherson, C. D., Cordes, A., Cremata, E. J., Penna, N. D., Estel, V., Fedor, A., Fitneva, S. A., Frank, M. C., Grange, J. A., Hartshorne, J. K., Hasselman, F., Henninger, F., ... Zuni, K. (2016). Response to comment on "Estimating the reproducibility of psychological science." Science, 351(6277), 1037-1037. https://doi.org/10.1126/science.aad9163

Anderson, S. F., \& Maxwell, S. E. (2017). Addressing the "replication crisis": Using original studies to design replication studies with appropriate statistical power. Multivariate Behavioral Research, 52(3), 305-324. https://doi.org/10.1080/00273171.2017.1289361 
Nuijten, M. B. (accepted chapter). Assessing and improving robustness of psychological research findings in four steps. In W. O'Donohue, A. Masuda, and S. Lilienfeld (Eds.), Clinical psychology and questionable research practices. Springer.

Appelbaum, M., Cooper, H., Kline, R. B., Mayo-Wilson, E., Nezu, A. M., \& Rao, S. M. (2018). Journal article reporting standards for quantitative research in psychology: The APA Publications and Communications Board task force report. American Psychologist, 73(1), 3. https://doi.org/10.1037/amp0000191

Association for Psychological Science. (n.d.). Registered Replication Reports. Association for Psychological Science - APS. Retrieved February 27, 2021, from https://www.psychologicalscience.org/publications/replication

Bakker, M., van Dijk, A., \& Wicherts, J. M. (2012). The rules of the game called psychological science. Perspectives on Psychological Science, 7(6), 543-554. https://doi.org/10.1177/1745691612459060

Bakker, M., \& Wicherts, J. M. (2011). The (mis)reporting of statistical results in psychology journals. Behavior Research Methods, 43(3), 666-678. https://doi.org/10.3758/s13428-011-0089-5 Bavel, J. J. V., Mende-Siedlecki, P., Brady, W. J., \& Reinero, D. A. (2016). Contextual sensitivity in scientific reproducibility. Proceedings of the National Academy of Sciences, 113(23), 64546459. https://doi.org/10.1073/pnas.1521897113

Benjamin, D. J., Berger, J. O., Johannesson, M., Nosek, B. A., Wagenmakers, E.-J., Berk, R., Bollen, K. A., Brembs, B., Brown, L., Camerer, C., Cesarini, D., Chambers, C. D., Clyde, M., Cook, T. D., De Boeck, P., Dienes, Z., Dreber, A., Easwaran, K., Efferson, C., ... Johnson, V. E. (2018). Redefine statistical significance. Nature Human Behaviour, 2(1), 6-10. https://doi.org/10.1038/s41562-017-0189-z

Brandt, M. J., IJzerman, H., Dijksterhuis, A., Farach, F. J., Geller, J., Giner-Sorolla, R., Grange, J. A., Perugini, M., Spies, J. R., \& van 't Veer, A. (2014). The replication recipe: What makes for a convincing replication? Journal of Experimental Social Psychology, 50, 217-224. https://doi.org/10.1016/j.jesp.2013.10.005 
Nuijten, M. B. (accepted chapter). Assessing and improving robustness of psychological research findings in four steps. In W. O'Donohue, A. Masuda, and S. Lilienfeld (Eds.), Clinical psychology and questionable research practices. Springer.

Brown, N. J. L., \& Heathers, J. A. J. (2017). The GRIM test: A simple technique detects numerous anomalies in the reporting of results in psychology. Social Psychological and Personality Science, 8(4), 363-369. https://doi.org/10.1177/1948550616673876

Button, K. S., loannidis, J. P. A., Mokrysz, C., Nosek, B. A., Flint, J., Robinson, E. S., \& Munafò, M. R. (2013). Power failure: Why small sample size undermines the reliability of neuroscience. Nature Reviews Neuroscience, 14(5), 365-376. https://doi.org/10.1038/nrn3475

Center for Open Science. (n.d.-a). Open Science Badges. Retrieved February 23, 2021, from https://www.cos.io/initiatives/badges

Center for Open Science. (n.d.-b). TOP Guidelines. Retrieved February 28, 2021, from https://www.cos.io/initiatives/top-guidelines

Chabris, C. F., Hebert, B. M., Benjamin, D. J., Beauchamp, J., Cesarini, D., van der Loos, M., Johannesson, M., Magnusson, P. K. E., Lichtenstein, P., Atwood, C. S., Freese, J., Hauser, T. S., Hauser, R. M., Christakis, N., \& Laibson, D. (2012). Most reported genetic associations with general intelligence are probably false positives. Psychological Science, 23(11), 1314-1323. https://doi.org/10.1177/0956797611435528

Chambers, C. D. (2013). Registered reports: A new publishing initiative at Cortex. Cortex, 49(3), 609610. https://doi.org/10.1016/j.cortex.2012.12.016

Chambers, C. D. (2017). The seven deadly sins of psychology: A manifesto for reforming the culture of scientific practice. Princeton University Press. https://doi.org/10.1515/9781400884940

Chambers, C. D. (2018). Introducing the transparency and openness promotion (TOP) guidelines and badges for open practices at Cortex. Cortex, 106, 316-318. https://doi.org/10.1016/j.cortex.2018.08.001

Chambers, C. D. (2020). Verification Reports: A new article type at Cortex. Cortex. https://doi.org/10.1016/j.cortex.2020.04.020 
Nuijten, M. B. (accepted chapter). Assessing and improving robustness of psychological research findings in four steps. In W. O'Donohue, A. Masuda, and S. Lilienfeld (Eds.), Clinical psychology and questionable research practices. Springer.

Christensen, G., Dafoe, A., Miguel, E., Moore, D. A., \& Rose, A. K. (2019). A study of the impact of data sharing on article citations using journal policies as a natural experiment. PLOS ONE, 14(12), e0225883. https://doi.org/10.1371/journal.pone.0225883

Cumming, G. (2014). The new statistics: Why and how. Psychological Science, 25(1), 7-29. https://doi.org/10.1177/0956797613504966

Dalen, H. P. van, \& Henkens, K. (2012). Intended and unintended consequences of a publish-orperish culture: A worldwide survey. Journal of the American Society for Information Science and Technology, 63(7), 1282-1293. https://doi.org/10.1002/asi.22636

Doyen, S., Klein, O., Pichon, C.-L., \& Cleeremans, A. (2012). Behavioral priming: It's all in the mind, but whose mind? PLOS ONE, 7(1), e29081. https://doi.org/10.1371/journal.pone.0029081

Epskamp, S., \& Nuijten, M. B. (2014). statcheck: Extract statistics from articles and recompute pvalues.

Eronen, M. I., \& Bringmann, L. F. (2021). The theory crisis in psychology: How to move forward. Perspectives on Psychological Science: A Journal of the Association for Psychological Science, 1745691620970586. https://doi.org/10.1177/1745691620970586

Etz, A., \& Vandekerckhove, J. (2016). A Bayesian perspective on the reproducibility project: Psychology. PloS One, 11(2), e0149794.

Fanelli, D. (2010). "Positive" results increase down the hierarchy of the sciences. PLOS ONE, 5(4), e10068. https://doi.org/10.1371/journal.pone.0010068

Fiedler, K., \& Schwarz, N. (2016). Questionable research practices revisited. Social Psychological and Personality Science, 7(1), 45-52. https://doi.org/10.1177/1948550615612150

Flake, J. K., \& Fried, E. I. (2020). Measurement schmeasurement: Questionable measurement practices and how to avoid them. Advances in Methods and Practices in Psychological Science, 2515245920952393. https://doi.org/10.1177/2515245920952393 
Nuijten, M. B. (accepted chapter). Assessing and improving robustness of psychological research findings in four steps. In W. O'Donohue, A. Masuda, and S. Lilienfeld (Eds.), Clinical psychology and questionable research practices. Springer.

Francis, G., Tanzman, J., \& Matthews, W. J. (2014). Excess success for psychology articles in the journal Science. PloS One, 9(12), e114255.

Franco, A., Malhotra, N., \& Simonovits, G. (2016). Underreporting in psychology experiments: Evidence from a study registry. Social Psychological and Personality Science, 7(1), 8-12. https://doi.org/10.1177/1948550615598377

Frank, M. C., \& Saxe, R. (2012). Teaching replication. Perspectives on Psychological Science, 7(6), 600-604. https://doi.org/10.1177/1745691612460686

Gelman, A., \& Loken, E. (2013). The garden of forking paths: Why multiple comparisons can be a problem, even when there is no "fishing expedition" or "p-hacking" and the research hypothesis was posited ahead of time. Department of Statistics, Columbia University, 348.

Georgescu, C., \& Wren, J. D. (2018). Algorithmic identification of discrepancies between published ratios and their reported confidence intervals and P-values. Bioinformatics, 34(10), 17581766. https://doi.org/10.1093/bioinformatics/btx811

Gilbert, D. T., King, G., Pettigrew, S., \& Wilson, T. D. (2016). Comment on “Estimating the reproducibility of psychological science." Science, 351(6277), 1037-1037. https://doi.org/10.1126/science.aad7243

Greenwald, A. G. (1975). Consequences of prejudice against the null hypothesis. Psychological Bulletin, 82(1), 1-20.

Hardwicke, T. E., Bohn, M., MacDonald, K., Hembacher, E., Nuijten, M. B., Peloquin, B. N., DeMayo, B. E., Long, B., Yoon, E. J., \& Frank, M. C. (2020). Analytic reproducibility in articles receiving open data badges at Psychological Science: An observational study. Preprint Retrieved from Https://Osf.lo/Preprints/Metaarxiv/H35wt/. https://doi.org/10.31222/osf.io/h35wt

Hardwicke, T. E., Mathur, M. B., MacDonald, K., Nilsonne, G., Banks, G. C., Kidwell, M. C., Hofelich Mohr, A., Clayton, E., Yoon, E. J., \& Henry Tessler, M. (2018). Data availability, reusability, and analytic reproducibility: Evaluating the impact of a mandatory open data policy at the 
Nuijten, M. B. (accepted chapter). Assessing and improving robustness of psychological research findings in four steps. In W. O'Donohue, A. Masuda, and S. Lilienfeld (Eds.), Clinical psychology and questionable research practices. Springer.

journal Cognition. Royal Society Open Science, 5(8), 180448.

https://doi.org/10.1098/rsos.180448

Horstmann, K. T., Arslan, R. C., \& Greiff, S. (2020). Generating codebooks to ensure the independent use of research data: Some guidelines. European Journal of Psychological Assessment, 36(5), 721-729. https://doi.org/10.1027/1015-5759/a000620

John, L. K., Loewenstein, G., \& Prelec, D. (2012). Measuring the prevalence of questionable research practices with incentives for truth telling. Psychological Science, 23(5), 524-532. https://doi.org/10.1177/0956797611430953

Jonas, K. J., Cesario, J., Alger, M., Bailey, A. H., Bombari, D., Carney, D., Dovidio, J. F., Duffy, S., Harder, J. A., Huistee, D. van, Jackson, B., Johnson, D. J., Keller, V. N., Klaschinski, L., LaBelle, O., LaFrance, M., Latu, I. M., Morssinkhoff, M., Nault, K., ... Tybur, J. M. (2017). Power poses - where do we stand? Comprehensive Results in Social Psychology, 2(1), 139-141. https://doi.org/10.1080/23743603.2017.1342447

Kerr, N. L. (1998). HARKing: Hypothesizing after the results are known. Personality and Social Psychology Review, 2(3), 196-217. https://doi.org/10.1207/s15327957pspr0203_4

Kidwell, M. C., Lazarević, L. B., Baranski, E., Hardwicke, T. E., Piechowski, S., Falkenberg, L.-S., Kennett, C., Slowik, A., Sonnleitner, C., \& Hess-Holden, C. (2016). Badges to acknowledge open practices: A simple, low-cost, effective method for increasing transparency. PLoS Biology, 14(5), e1002456. https://doi.org/10.1371/journal.pbio.1002456

Klein, O., Hardwicke, T. E., Aust, F., Breuer, J., Danielsson, H., Hofelich Mohr, A., IJzerman, H., Nilsonne, G., Vanpaemel, W., \& Frank, M. C. (2018). A practical guide for transparency in psychological science. Collabra: Psychology, 4(1), 1-15. https://doi.org/10.1525/collabra.158 Klein, R. A., Ratliff, K. A., Vianello, M., Adams Jr, R. B., Bahník, Š., Bernstein, M. J., Bocian, K., Brandt, M. J., Brooks, B., \& Brumbaugh, C. C. (2014). Investigating variation in replicability: A "Many Labs" replication project. Social Psychology. 
Nuijten, M. B. (accepted chapter). Assessing and improving robustness of psychological research findings in four steps. In W. O'Donohue, A. Masuda, and S. Lilienfeld (Eds.), Clinical psychology and questionable research practices. Springer.

Kochari, A. R., \& Ostarek, M. (2018). Introducing a replication-first rule for PhD projects (commmentary on Zwaan et al., 'Making replication mainstream'). Behavioral and Brain Sciences, 41. https://doi.org/10.1017/S0140525X18000730

Lakatos, I., \& Musgrave, A. (1970). Criticism and the growth of knowledge. Cambridge University Press.

Lakens, D., Adolfi, F. G., Albers, C. J., Anvari, F., Apps, M. A. J., Argamon, S. E., Baguley, T., Becker, R. B., Benning, S. D., Bradford, D. E., Buchanan, E. M., Caldwell, A. R., Van Calster, B., Carlsson, R., Chen, S.-C., Chung, B., Colling, L. J., Collins, G. S., Crook, Z., ... Zwaan, R. A. (2018). Justify your alpha. Nature Human Behaviour, 2(3), 168-171. https://doi.org/10.1038/s41562-0180311-x

Lakens, D., \& Evers, E. R. K. (2014). Sailing From the Seas of Chaos Into the Corridor of Stability: Practical Recommendations to Increase the Informational Value of Studies. Perspectives on Psychological Science, 9(3), 278-292. https://doi.org/10.1177/1745691614528520

LeBel, E. P. (2015). A new replication norm for psychology. Collabra, 1(4). https://doi.org/10.1525/collabra.23

LeBel, E. P., Berger, D., Campbell, L., \& Loving, T. J. (2017). Falsifiability is not optional. Journal of Personality and Social Psychology, 113(2), 254-261. https://doi.org/10.1037/pspi0000106 LeBel, E. P., \& Campbell, L. (2013). Heightened sensitivity to temperature cues in individuals with high anxious attachment: Real or elusive phenomenon? Psychological Science, 24(10), 21282130. https://doi.org/10.1177/0956797613486983

LeBel, E. P., McCarthy, R. J., Earp, B. D., Elson, M., \& Vanpaemel, W. (2018). A unified framework to quantify the credibility of scientific findings. Advances in Methods and Practices in Psychological Science, 1(3), 389-402. https://doi.org/10.1177/2515245918787489

LeBel, E. P., Vanpaemel, W., Cheung, I., \& Campbell, L. (2019). A brief guide to evaluate replications. Meta-Psychology, 3. https://doi.org/10.15626/MP.2018.843 
Nuijten, M. B. (accepted chapter). Assessing and improving robustness of psychological research findings in four steps. In W. O'Donohue, A. Masuda, and S. Lilienfeld (Eds.), Clinical psychology and questionable research practices. Springer.

Makel, M. C., Plucker, J. A., \& Hegarty, B. (2012). Replications in psychology research: How often do they really occur? Perspectives on Psychological Science, 7(6), 537-542. https://doi.org/10.1177/1745691612460688

ManyBabies Consortium. (2020). Quantifying sources of variability in infancy research using the infant-directed-speech preference. Advances in Methods and Practices in Psychological Science, 3(1), 24-52. https://doi.org/10/ghwq2t

Marsman, M., \& Wagenmakers, E.-J. (2017). Bayesian benefits with JASP. European Journal of Developmental Psychology, 14(5), 545-555. https://doi.org/10.1080/17405629.2016.1259614

Matthews, W. J. (2012). How much do incidental values affect the judgment of time? Psychological Science, 23(11), 1432-1434. https://doi.org/10.1177/0956797612441609

Mayo, D. G. (2018). Statistical inference as severe testing. https://www.cambridge.org/core/books/statistical-inference-as-severe-testing/copyrightpage/55AF1D228E1401D0912B1D59E7400BD3

Meehl, P. E. (1990). Appraising and amending theories: The strategy of lakatosian defense and two principles that warrant it. Psychological Inquiry, 1(2), 108-141. https://doi.org/10.1207/s15327965pli0102_1

Morling, B. (2020). Research methods in psychology (4th ed.). W. W. Norton \& Company. Moshontz, H., Campbell, L., Ebersole, C. R., IJzerman, H., Urry, H. L., Forscher, P. S., Grahe, J. E., McCarthy, R. J., Musser, E. D., Antfolk, J., Castille, C. M., Evans, T. R., Fiedler, S., Flake, J. K., Forero, D. A., Janssen, S. M. J., Keene, J. R., Protzko, J., Aczel, B., ... Chartier, C. R. (2018). The Psychological Science Accelerator: Advancing psychology through a distributed collaborative network. Advances in Methods and Practices in Psychological Science, 1(4), 501-515. https://doi.org/10.1177/2515245918797607 
Nuijten, M. B. (accepted chapter). Assessing and improving robustness of psychological research findings in four steps. In W. O'Donohue, A. Masuda, and S. Lilienfeld (Eds.), Clinical psychology and questionable research practices. Springer.

Munafò, M. R., Nosek, B. A., Bishop, D. V., Button, K. S., Chambers, C. D., Du Sert, N. P., Simonsohn, U., Wagenmakers, E.-J., Ware, J. J., \& loannidis, J. P. A. (2017). A manifesto for reproducible science. Nature Human Behaviour, 1(1), 1-9. https://doi.org/10.1038/s41562-016-0021

Naudet, F., Sakarovitch, C., Janiaud, P., Cristea, I., Fanelli, D., Moher, D., \& loannidis, J. P. A. (2018). Data sharing and reanalysis of randomized controlled trials in leading biomedical journals with a full data sharing policy: Survey of studies published in The BMJ and PLOS Medicine. British Medical Journal, 360, k400. https://doi.org/10.1136/bmj.k400

Neuliep, J. W., \& Crandall, R. (1993). Everyone was wrong: There are lots of replications out there. Journal of Social Behavior and Personality, 8(6), 1-8.

Nieuwenhuis, S., Forstmann, B. U., \& Wagenmakers, E.-J. (2011). Erroneous analyses of interactions in neuroscience: A problem of significance. Nature Neuroscience, 14(9), 1105-1107. https://doi.org/10.1038/nn.2886

Nosek, B. A., Hardwicke, T. E., Moshontz, H., Allard, A., Corker, K. S., Almenberg, A. D., Fidler, F., Hilgard, J., Kline, M., Nuijten, M. B., Rohrer, J. M., Romero, F., Scheel, A. M., Scherer, L., Schönbrodt, F., \& Vazire, S. (2021). Replicability, robustness, and reproducibility in psychological science. PsyArXiv. https://doi.org/10.31234/osf.io/ksfvq

Nosek, B. A., \& Lakens, D. (2014). Registered reports: A method to increase the credibility of published results. Social Psychology, 45(3), 137-141. https://doi.org/10.1027/18649335/a000192

Nosek, B. A., Spies, J. R., \& Motyl, M. (2012). Scientific Utopia: II. Restructuring incentives and practices to promote truth over publishability. Perspectives on Psychological Science, 7(6), 615-631. https://doi.org/10.1177/1745691612459058

Nuijten, M. B., Bakker, M., Maassen, E., \& Wicherts, J. M. (2018). Verify original results through reanalysis before replicating. Behavioral and Brain Sciences, 41, e143.

https://doi.org/10.1017/\$0140525X18000791 
Nuijten, M. B. (accepted chapter). Assessing and improving robustness of psychological research findings in four steps. In W. O'Donohue, A. Masuda, and S. Lilienfeld (Eds.), Clinical psychology and questionable research practices. Springer.

Nuijten, M. B., Borghuis, J., Veldkamp, C. L., Dominguez-Alvarez, L., Van Assen, M. A., \& Wicherts, J. M. (2017). Journal data sharing policies and statistical reporting inconsistencies in psychology. Collabra: Psychology, 3(1). https://doi.org/10.1525/collabra.102

Nuijten, M. B., Hartgerink, C. H. J., van Assen, M. A. L. M., Epskamp, S., \& Wicherts, J. M. (2016). The prevalence of statistical reporting errors in psychology (1985-2013). Behavior Research Methods, 48(4), 1205-1226. https://doi.org/10.3758/s13428-015-0664-2

Nuijten, M. B., Van Assen, M. A. L. M., Hartgerink, C. H. J., Epskamp, S., \& Wicherts, J. (2017). The validity of the tool "statcheck" in discovering statistical reporting inconsistencies. PsyArXiv. https://doi.org/10.31234/osf.io/tcxaj

O'Donohue, W. (2021). Some Popperian notes regarding replication failures in psychology. Journal of Theoretical and Philosophical Psychology.

Open Science Collaboration. (2012). An open, large-scale, collaborative effort to estimate the reproducibility of psychological science. Perspectives on Psychological Science, 7(6), 657660. https://doi.org/10.1177/1745691612462588

Open Science Collaboration. (2015). Estimating the reproducibility of psychological science. Science, 349(6251), aac4716. https://doi.org/10.1126/science.aac4716

Pashler, H., \& Harris, C. R. (2012). Is the replicability crisis overblown? Three arguments examined. Perspectives on Psychological Science, 7(6), 531-536. https://doi.org/10.1177/1745691612463401

Pashler, H., Rohrer, D., \& Harris, C. R. (2013). Can the goal of honesty be primed? Journal of Experimental Social Psychology, 49(6), 959-964. https://doi.org/10.1016/j.jesp.2013.05.011 Pashler, H., \& Wagenmakers, E. (2012). Editors' introduction to the special section on replicability in psychological science: A crisis of confidence? Perspectives on Psychological Science, 7(6), 528-530. https://doi.org/10.1177/1745691612465253 
Nuijten, M. B. (accepted chapter). Assessing and improving robustness of psychological research findings in four steps. In W. O'Donohue, A. Masuda, and S. Lilienfeld (Eds.), Clinical psychology and questionable research practices. Springer.

Patel, C. J., Burford, B., \& loannidis, J. P. A. (2015). Assessment of vibration of effects due to model specification can demonstrate the instability of observational associations. Journal of Clinical Epidemiology, 68(9), 1046-1058.

Peng, R. D. (2011). Reproducible research in computational science. Science, 334(6060), 1226-1227. https://doi.org/10.1126/science.1213847

Petrocelli, J. V., Clarkson, J. J., Whitmire, M. B., \& Moon, P. E. (2013). When $a b \neq c-c^{\prime}$ : Published errors in the reports of single-mediator models. Behavior Research Methods, 45(2), 595-601. https://doi.org/10.3758/s13428-012-0262-5

Piwowar, H. A., Day, R. S., \& Fridsma, D. B. (2007). Sharing detailed research data is associated with increased citation rate. PLOS ONE, 2(3), e308. https://doi.org/10.1371/journal.pone.0000308

Popper, K. R. (1959). The logic of scientific discovery. University Press.

Rife, S. C., Nuijten, M. B., \& Epskamp, S. (2016). statcheck: Extract statistics from articles and recompute -values [web application]. http://statcheck.io

Silberzahn, R., UhImann, E. L., Martin, D. P., Anselmi, P., Aust, F., Awtrey, E., Bahník, Š., Bai, F., Bannard, C., Bonnier, E., Carlsson, R., Cheung, F., Christensen, G., Clay, R., Craig, M. A., Dalla Rosa, A., Dam, L., Evans, M. H., Flores Cervantes, I., ... Nosek, B. A. (2018). Many analysts, one data set: Making transparent how variations in analytic choices affect results. Advances in Methods and Practices in Psychological Science, 1(3), 337-356. https://doi.org/10.1177/2515245917747646

Simmons, J. P., Nelson, L. D., \& Simonsohn, U. (2011). False-positive psychology: Undisclosed flexibility in data collection and analysis allows presenting anything as significant. Psychological Science, 22(11), 1359-1366. https://doi.org/10.1177/0956797611417632

Simonsohn, U. (2015). Small telescopes: Detectability and the evaluation of replication results. Psychological Science, 26(5), 559-569. https://doi.org/10.1177/0956797614567341 
Nuijten, M. B. (accepted chapter). Assessing and improving robustness of psychological research findings in four steps. In W. O'Donohue, A. Masuda, and S. Lilienfeld (Eds.), Clinical psychology and questionable research practices. Springer.

Simonsohn, U., Simmons, J. P., \& Nelson, L. D. (2019). Specification curve: Descriptive and inferential statistics on all reasonable specifications (SSRN Scholarly Paper ID 2694998). Social Science Research Network. https://doi.org/10.2139/ssrn.2694998

Stark, P. B. (2018). Before reproducibility must come preproducibility. Nature, 557(7707), 613-613. https://doi.org/10.1038/d41586-018-05256-0

Steegen, S., Tuerlinckx, F., Gelman, A., \& Vanpaemel, W. (2016). Increasing transparency through a multiverse analysis. Perspectives on Psychological Science, 11(5), 702-712. https://doi.org/10.1177/1745691616658637

Sterling, T. D. (1959). Publication decisions and their possible effects on inferences drawn from tests of significance-Or vice versa. Journal of the American Statistical Association, 54(285), 3034. https://doi.org/10.1080/01621459.1959.10501497

Sterling, T. D., Rosenbaum, W. L., \& Weinkam, J. J. (1995). Publication decisions revisited: The effect of the outcome of statistical tests on the decision to publish and vice versa. The American Statistician, 49(1), 108-112. https://doi.org/10.1080/00031305.1995.10476125

Stodden, V. C. (2010). Reproducible research: Addressing the need for data and code sharing in computational science.

Sweeney, L. (2002). K-anonymity: A model for protecting privacy. International Journal of Uncertainty, Fuzziness and Knowledge-Based Systems, 10(05), 557-570. https://doi.org/10.1142/S0218488502001648

The Dutch Research Council. (n.d.). Replication studies / NWO. Retrieved February 24, 2021, from https://www.nwo.nl/en/researchprogrammes/replication-studies

Tijdink, J. K., Verbeke, R., \& Smulders, Y. M. (2014). Publication pressure and scientific misconduct in medical scientists. Journal of Empirical Research on Human Research Ethics, 9(5), 64-71. https://doi.org/10.1177/1556264614552421 
Nuijten, M. B. (accepted chapter). Assessing and improving robustness of psychological research findings in four steps. In W. O'Donohue, A. Masuda, and S. Lilienfeld (Eds.), Clinical psychology and questionable research practices. Springer.

van Aert, R. C. M., Nuijten, M. B., Olsson-Collentine, A., Stoevenbelt, A. H., Van den Akker, O. R., \& Wicherts, J. M. (2021). Comparing the prevalence of statistical reporting inconsistencies in COVID-19 preprints and matched controls: A Registered Report. Royal Society Open Science. https://doi.org/10.17605/OSF.IO/WCND4

Verhagen, J., \& Wagenmakers, E.-J. (2014). Bayesian tests to quantify the result of a replication attempt. Journal of Experimental Psychology: General, 143(4), 1457.

Wagenmakers, E.-J., Wetzels, R., Borsboom, D., van der Maas, H. L. J., \& Kievit, R. A. (2012). An agenda for purely confirmatory research. Perspectives on Psychological Science, 7(6), 632638. https://doi.org/10.1177/1745691612463078

Wicherts, J. M. (2013). Science revolves around the data. Journal of Open Psychology Data, 1(1), e1. https://doi.org/10.5334/jopd.e1

Wicherts, J. M., Borsboom, D., Kats, J., \& Molenaar, D. (2006). The poor availability of psychological research data for reanalysis. American Psychologist, 61(7), 726. https://doi.org/10.1037/0003-066X.61.7.726

Wicherts, J. M., Kievit, R. A., Bakker, M., \& Borsboom, D. (2012). Letting the daylight in: Reviewing the reviewers and other ways to maximize transparency in science. Frontiers in Computational Neuroscience, 6. https://doi.org/10.3389/fncom.2012.00020

Wilkinson, M. D., Dumontier, M., Aalbersberg, Ij. J., Appleton, G., Axton, M., Baak, A., Blomberg, N., Boiten, J.-W., da Silva Santos, L. B., Bourne, P. E., Bouwman, J., Brookes, A. J., Clark, T., Crosas, M., Dillo, I., Dumon, O., Edmunds, S., Evelo, C. T., Finkers, R., ... Mons, B. (2016). The FAIR Guiding Principles for scientific data management and stewardship. Scientific Data, 3(1), 160018. https://doi.org/10.1038/sdata.2016.18

Zwaan, R., Etz, A., Lucas, R., \& Donnellan, B. (2017). Making replication mainstream. Behavioral and Brain Sciences: An International Journal of Current Research and Theory with Open Peer Commentary, 1-50. https://doi.org/10.1017/S0140525X17001972 
Nuijten, M. B. (accepted chapter). Assessing and improving robustness of psychological research findings in four steps. In W. O'Donohue, A. Masuda, and S. Lilienfeld (Eds.), Clinical psychology and questionable research practices. Springer. 